\title{
Visible light spectroscopic analysis of Methylene Blue in water; the resonance virtual equilibrium hypothesis
}

Amparo Fernández-Pérez, Teresa Valdés-Solís and Gregorio Marbán ${ }^{1}$

INCAR-CSIC - c/Francisco Pintado Fe 26, 33011 Oviedo (Spain). Tel. +34 985119090

\begin{abstract}
After a critical approach to the centennial literature on Methylene Blue self-aggregation in water, accurate visible-light absorption spectra and thermodynamic equilibrium constants for the monomeric and dimeric forms of this dye in water at different temperatures were obtained by means of a novel procedure based on the Beer-Lambert equation. A thermo-solvatochromic effect was clearly detected in the monomer spectra, which we believe to be the main source of inaccuracy in all previous works. The only published explanation for this phenomenon, the existence of a colorless form of the monomer, is refuted here. Instead, a new notion has been introduced; the resonance virtual equilibrium hypothesis. According to this hypothesis the temperature-dependent absorption behavior of the monomer is provoked by the change in its electron charge distribution with the variation in the temperaturedependent dielectric constant of water. The monomer charge distribution stands between those of the virtual resonance forms, being the absorption spectrum of the monomer a composition of the theoretical spectra for the virtual mesomers, whose proportion is established by a temperature-dependent equilibrium constant. The application of this hypothesis allowed the virtual equilibrium constants and spectra for the mesomers to be evaluated with remarkable accuracy.
\end{abstract}

KEYWORDS: Methylene Blue; Uv-Visible spectrophotometry; equilibrium constant; thermosolvatochromism; self-aggregation; resonance form

\footnotetext{
${ }^{1}$ Corresponding Author: e-mail: greca@ incar.csic.es (G. Marbán)
} 


\section{INTRODUCTION}

Concentration-dependent metachromasy of aqueous Methylene Blue (MB) solutions is well known and can be related to the formation of aggregates [1-4]. At low MB concentration (below 20 ppm, according to some authors [5], or $~ 70 \mathrm{ppm}$, according to others [6]) the existence of aggregation beyond the dimeric form of the dye (i.e. trimeric or tetrameric forms) can be neglected. Within this concentration range, object of the present work, the specific molar absorptivity of the dimer affects the shape of the Uv-Vis spectra according to its ratio in the dimer/monomer mixture. The commonly accepted existence of this effect is only challenged by the group of González-Aguilar [7], although their calculations have been questioned in [8]. In aqueous MB solutions, monomer molecules and dimers coexist at ambient temperature, both showing fairly different absorption spectra [9, 10]. The concentration of the dimeric form increases with the total concentration of MB molecules due to the specific form of the reaction constant for the monomer/dimer equilibrium. Such equilibrium has been studied since long and a variety of thermodynamic (entropy and enthalpy of dimerization) and optical (molar attenuation coefficients) data are available in literature [1, 6, 9, 11-18]. These data were obtained by means of a methodology firstly applied by Rabinowitch and Epstein [11], which consists of solving the equations that link the absorbance with the monomer and dimer concentrations at two or several wavelengths to derive the molar attenuation coefficients for the monomer and dimer, either via extrapolation techniques $[1,6,12-14]$ or by the error minimization $[9,15]$. However, in spite of the long time that the scientific community has dedicated to this subject, the equilibrium constants and optical parameters have always been evaluated with a high degree of uncertainty (Table 1), which makes us believe that the cause of such inaccuracy has escaped the attention of the scientists to date. The present work proves that the reason for the uncertainty lies in a common and erroneous assumption since the time of Rabinowitch and Epstein [11] for the calculation of the aggregation degree and the dimerization 
constant. This assumption is that the absorption spectrum of the monomer is independent of the temperature (invariant-monomer assumption from now on). However, this universally employed assumption is in contradiction with the long known fact that the maximum attenuation coefficient of the monomer spectrum (obtained via extrapolation at a very low MB concentration) shows a slight decrease at increasing temperatures [12] (see Table 1). The authors that detected this trend, Lewis et al. [12], proposed as an explanation the existence in solution of a "considerable" amount of a colorless form of the MB monomer. According to them, this form could be produced by the hydrolytic addition of water to the cationic monomer, process that they found surprisingly favored at increasing temperatures (the colorless species might be similar to the Leuco-Methylene Blue molecule, which is formed by the reduction of monomeric MB in the absence of oxygen [19]). To our knowledge, no more studies about the effect of temperature on the light absorption by the MB monomer in water are available in literature.

In the present work we have employed in a novel manner the old and simple equations used by most authors for nearly a century, with two main objectives; (i) to evaluate reliable and accurate optical and thermodynamic parameters for MB in water solutions at low concentration, and (ii) to shed fresh light on the spectroscopic behavior of the MB monomer in water at varying temperatures, that calls into question not only the invariant-monomer assumption but also the hypothesis by Lewis et al. [12].

\section{EXPERIMENTAL}

The absorption spectra (400-800 $\mathrm{nm}$ at $1 \mathrm{~nm}$ step) of 6 MB (C.I. 52015; analytical grade) solutions in deionized water in the $1.1 \times 10^{-6}-3.4 \times 10^{-5} \mathrm{~mol} \mathrm{~L}^{-1}(0.35-11 \mathrm{ppm})$ concentration range were obtained at temperatures in the $9-70^{\circ} \mathrm{C}$ range using an Uv-Vis spectrometer (Shimazdu UV-2401PC) with an Uv quartz cuvette of $700 \mu \mathrm{L}$ volume and $1 \mathrm{~cm}$ path length. The temperature of the cell was kept constant 
using a Lauda Alpha RA8 thermo-circulating bath. Every measure was repeated thrice. Ad hoc Microsoft $^{\circledR}$ Excel macros were designed to perform the error minimization tasks, incorporating the Solver complement of Microsoft ${ }^{\circledR}$ Excel configured to employ the Generalized Reduced Gradient algorithm with central derivatives.

\section{HISTORICAL BACKGROUND}

Application of the Beer-Lambert equation to the absorption spectrum of Methylene Blue in water at a given wavelength yields:

$A_{\lambda}=\varepsilon_{\lambda} L C_{M B}=A_{\lambda}^{m}+A_{\lambda}^{d}=\varepsilon_{\lambda}^{m} L C_{m}+\varepsilon_{\lambda}^{d} L C_{d}$

where $A_{\lambda}$ is the absorbance at the $\lambda$ wavelength, $\varepsilon_{\lambda}, \varepsilon_{\lambda}^{m}$ and $\varepsilon_{\lambda}^{d}$ are the molar attenuation coefficients of the solution, the monomer and the dimer, respectively, at the same wavelength $\left(\mathrm{L} \mathrm{mol}^{-1} \mathrm{~cm}^{-1}\right), L$ is the path length $(\mathrm{cm})$ and $C_{M B}, C_{m}$ and $C_{d}$ are the $\mathrm{MB}$, monomer and dimer concentrations, respectively (mol L-1). The total MB concentration can be evaluated from $C_{m}$ and $C_{d}$ as:

$C_{M B}=C_{m}+2 C_{d}$

The objective of all calculation methods has been to evaluate the molar attenuation coefficients for the monomer and the dimer, from which the aggregation degree can be readily calculated. In the pioneering work by Rabinowitch and Epstein [11], the molar attenuation coefficients for the MB dimer and monomer at two wavelengths $(656.5$ and $600 \mathrm{~nm})$ were evaluated by extrapolation to $C_{M B}=0$ and $C_{M B}=\infty$ of the observed molar attenuation coefficients. Then the fraction of monomer was calculated by solving the following equation

$X_{m}=\frac{C_{m}}{C_{M B}}=\frac{\varepsilon_{\lambda}-0.5 \varepsilon_{\lambda}^{d}}{\varepsilon_{\lambda}^{m}-0.5 \varepsilon_{\lambda}^{d}}$ 
where $\varepsilon_{\lambda}$ is the observed molar attenuation coefficient at $\lambda$. This equation directly derives from equation (1), and the value of $K_{d}$ can be subsequently obtained as

$K_{d}=\frac{C_{d}}{C_{m}^{2}}=\frac{1-X_{m}}{2 C_{M B} X_{m}^{2}}$

The values of the so obtained molar attenuation coefficients are indicated in Table 1, together with those obtained by other authors and, when available, the derived thermodynamic parameters. Bergmann and O'Konski [6] turned the method of Rabinowitch and Epstein upside down by firstly assuming a certain value of $K_{d}$ from which they evaluated $X_{m}$ at different values of $C_{M B}$ via equation (4). Then, they represented the values of $X_{m}$ versus the observed molar attenuation coefficients and obtained the values of $\varepsilon_{\lambda}^{m}$ and $\varepsilon_{\lambda}^{d}$ by extrapolation at $X_{m}=1$ and $X_{m}=0$, respectively [equation (3)]. The value of $K_{d}$ was varied until attaining coefficients of determination for equation (4) closest to 1 for several $\lambda$ values. Although the molar attenuation coefficients could be determined by this method, the authors themselves acknowledged that it was not sensitive enough to distinguish between several equilibrium constants. Gosh [13] used the Rabinowitch and Epstein method but with the condition that the molar attenuation coefficients of all the aggregates had to be the same at the wavelength for maximum absorbance in the monomer spectrum, assumption that can in no way be justified. Another typical extrapolation technique is to assume that $C_{M B} \approx C_{m}$ and $C_{M B} \approx C_{d}$ at a low and a at a high dye concentration, respectively [12]. This assumption may work fairly well for the monomer attenuation coefficients, but it is inadequate for the dimer, since a high increase in the MB concentration also produces increasingly complex polymers $[1]$.

The extrapolation methods produce large errors in the degree of association from only small differences in the molar attenuation coefficients [1] and the evaluation of the molar attenuation coefficient for the dimer is especially subjected to error [11]. With the advent of modern computers, the error minimization 
methods were introduced in an attempt to solve this problem [9, 15]. Spencer and Sutter [15] combined equations (1), (2) and (4) to obtain the following equation:

$A_{\lambda}=\left(\varepsilon_{\lambda}^{m}-\frac{\varepsilon_{\lambda}^{d}}{2}\right) L \frac{\sqrt{1+8 K_{d} C_{M B}}-1}{4 K_{d}}+\varepsilon_{\lambda}^{d} L \frac{C_{M B}}{2}$

Note that equation (5) here is different from equation (3) in [15], probably due to a transcription error in the work referred to. The minimization of the sum of squared deviations of experimental from calculated [by, hopefully, equation (5)] absorbances gave the values for the parameters at 610 and 660 $\mathrm{nm}$ indicated in Table 1. As can be seen in the table, some of the parameters evaluated by this method $\left(\varepsilon_{\lambda_{1}}^{d}, K_{d}, \Delta H_{d}\right.$ and $\left.\Delta S_{d}\right)$ suffer from a high dispersion (especially $\varepsilon_{\lambda_{1}}^{d}$ ), which makes it difficult to have an accurate evaluation of the aggregation degree. Furthermore, contrary to their own claim, the values of $\Delta H_{d}$ and $\Delta S_{d}$ (enthalpy and entropy of dimerization) obtained by the authors are much higher (in absolute values) than those obtained by a variety of investigators (Table 1). Yazdani et al. [9] employed a more complex methodology based on the construction of matrices including the absorbances in the whole wavelength range at different temperatures and $\mathrm{MB}$ concentrations. With this technique, the dimer spectrum, the monomer concentration profile and the equilibrium constant were evaluated by imposing three constrains; (i) the spectrum of the monomer, which is measured separately, (ii) the total concentration of the dye [equation (2)] and (iii) the thermodynamic relation between the temperature and the equilibrium constant (van't Hoff equation). As in [15], the sum of squared differences between experimental and predicted absorbances was used as a goodness of fit criterion. In spite of the complexity of the model, the results were far from being free of dispersion, as can be observed in Table 1 (see footnotes), and the dimer contribution to the characteristic peak of the monomer ( $\varepsilon_{\lambda_{1}}^{d}$ in Table 1$)$, evaluated from Figure 3-1b in [9], resulted to be much higher than that found by the rest of authors. In 
the following section a new error minimization method will be proposed. In spite of the simplicity of the method, it allows the optical and thermodynamic parameters to be accurately evaluated.

\section{METHOD FOR LOW MB CONCENTRATION}

At low MB concentrations (below $\sim 20 \mathrm{ppm}$, according to some authors [5], or $\sim 70 \mathrm{ppm}$, according to others [6]) the existence of the trimeric or tetrameric forms of the dye in solution can be neglected. Under this circumstance, the method proposed here simply consists of using the Beer-Lambert equation at two different wavelengths of the spectrum $\left(\lambda_{1}\right.$ and $\left.\lambda_{2}\right)$ :

$A_{\lambda_{1}}=\varepsilon_{\lambda_{1}} L C_{M B}=A_{\lambda_{1}}^{m}+A_{\lambda_{1}}^{d}=\varepsilon_{\lambda_{1}}^{m} L C_{m}+\varepsilon_{\lambda_{1}}^{d} L C_{d}$

$A_{\lambda_{2}}=\varepsilon_{\lambda_{2}} L C_{M B}=A_{\lambda_{2}}^{m}+A_{\lambda_{2}}^{d}=\varepsilon_{\lambda_{2}}^{m} L C_{m}+\varepsilon_{\lambda_{2}}^{d} L C_{d}$

In these equations, the molar attenuation coefficients are independent of temperature under the invariant-monomer assumption. It will be proven in the Results and discussion section that this assumption must be qualified. $\lambda_{1}$ and $\lambda_{2}$ should be close to the wavelengths at maximum absorbance for the monomer and the dimer, 664 and $610 \mathrm{~nm}$, respectively [9]. From equations (2), (6) and (7) the following equations are easily obtained:

$$
\begin{gathered}
C_{M B}=\alpha_{1} A_{\lambda_{1}}+\alpha_{2} A_{\lambda_{2}} \\
C_{m}=\beta_{1} A_{\lambda_{1}}+\beta_{2} A_{\lambda_{2}}
\end{gathered}
$$

in which $\alpha_{1}, \alpha_{2}, \beta_{1}$ and $\beta_{2}\left(\mathrm{~mol} \mathrm{~L}^{-1}\right)$ are defined as:

$$
\alpha_{1}=\frac{-2 \varepsilon_{\lambda_{2}}^{m}+\varepsilon_{\lambda_{2}}^{d}}{\left(\varepsilon_{\lambda_{1}}^{m} \varepsilon_{\lambda_{2}}^{d}-\varepsilon_{\lambda_{2}}^{m} \varepsilon_{\lambda_{1}}^{d}\right) L}
$$


$\alpha_{2}=\frac{2 \varepsilon_{\lambda_{1}}^{m}-\varepsilon_{\lambda_{1}}^{d}}{\left(\varepsilon_{\lambda_{1}}^{m} \varepsilon_{\lambda_{2}}^{d}-\varepsilon_{\lambda_{2}}^{m} \varepsilon_{\lambda_{1}}^{d}\right) L}$

$\beta_{1}=\frac{\varepsilon_{\lambda_{2}}^{d}}{\left(\varepsilon_{\lambda_{1}}^{m} \varepsilon_{\lambda_{2}}^{d}-\varepsilon_{\lambda_{2}}^{m} \varepsilon_{\lambda_{1}}^{d}\right) L}$

$\beta_{2}=\frac{-\varepsilon_{\lambda_{1}}^{d}}{\left(\varepsilon_{\lambda_{1}}^{m} \varepsilon_{\lambda_{2}}^{d}-\varepsilon_{\lambda_{2}}^{m} \varepsilon_{\lambda_{1}}^{d}\right) L}$

From these parameters, the molar attenuation coefficients can be evaluated as:

$\varepsilon_{\lambda_{1}}^{m}=\frac{\beta_{2}-\alpha_{2}}{\left(\alpha_{1} \beta_{2}-\alpha_{2} \beta_{1}\right) L}$

$\varepsilon_{\lambda_{2}}^{m}=\frac{\alpha_{1}-\beta_{1}}{\left(\alpha_{1} \beta_{2}-\alpha_{2} \beta_{1}\right) L}$

$\varepsilon_{\lambda_{1}}^{d}=\frac{-2 \beta_{1}}{\left(\alpha_{1} \beta_{2}-\alpha_{2} \beta_{1}\right) L}$

$\varepsilon_{\lambda_{2}}^{d}=\frac{2 \beta_{2}}{\left(\alpha_{1} \beta_{2}-\alpha_{2} \beta_{1}\right) L}$

Equations (10) and (11) resemble the elements of the inverse $\boldsymbol{R}$ rotation matrix equation by Yazdani et al. [9], this being the only similarity with the present method, which, as will be seen, yields optical and thermodynamic values that differ greatly from those obtained by these authors. $C_{M B}$ can be also expressed according to the following equation, derived from equations (2), (4) and (9):

$C_{M B}=\left(\beta_{1} A_{\lambda_{1}}+\beta_{2} A_{\lambda_{2}}\right)\left[1+2 K_{d}(T) \times\left(\beta_{1} A_{\lambda_{1}}+\beta_{2} A_{\lambda_{2}}\right)\right]$

Constant $K_{d}\left(\mathrm{~L} \mathrm{~mol}^{-1}\right)$ may be expressed either as a function of $A_{\lambda_{1}}$ and $A_{\lambda_{2}}$ or as a function of the temperature (van't Hoff equation):

$$
\begin{aligned}
& K_{d}\left(A_{\lambda}\right)=\frac{C_{M B}-\left(\beta_{1} A_{\lambda_{1}}+\beta_{2} A_{\lambda_{2}}\right)}{2 \times\left(\beta_{1} A_{\lambda_{1}}+\beta_{2} A_{\lambda_{2}}\right)^{2}} \\
& K_{d}(T)=e^{\left(\frac{\Delta S_{d}}{R}-\frac{\Delta H_{d}}{R T}\right)}
\end{aligned}
$$


where $\Delta S_{d}$ and $\Delta H_{d}$ are the enthalpy and entropy of dimerization, respectively. In order to determine the values of $\alpha_{1}, \alpha_{2}, \beta_{1}$ and $\beta_{2}$ and the thermodynamic parameters [ $\Delta S_{d}$ and $\Delta H_{d}$ ], the following mean square error function can be minimized for the $N \times M$ values of $A_{\lambda_{1}}$ and $A_{\lambda_{2}}$ obtained at different dye concentrations and temperatures:

$$
\begin{aligned}
& E_{T}=\frac{1}{N \times M} \sum\left\{C_{M B}-\left(\alpha_{1}(T) A_{\lambda_{1}}+\alpha_{2}(T) A_{\lambda_{2}}\right)\right\}^{2}+ \\
& +\frac{1}{N \times M} \sum\left\{C_{M B}-\left(\beta_{1}(T) A_{\lambda_{1}}+\beta_{2}(T) A_{\lambda_{2}}\right)\left[1+2 K_{d}(T) \times\left(\beta_{1}(T) A_{\lambda_{1}}+\beta_{2}(T) A_{\lambda_{2}}\right)\right]\right\}^{2}
\end{aligned}
$$

As already commented, under the invariant monomer assumption $\alpha_{1}, \alpha_{2}, \beta_{1}$ and $\beta_{2}$ parameters should be independent of the temperature [eqs. (10) to (13)]. However, as will be proven in the Results and discussion section, a clear temperature-dependence is observed in these parameters, as result of which they are expressed as functions of the temperature in equation (21). It has been also noticed that slight variations in the values of $\alpha_{1}(T), \alpha_{2}(T), \beta_{1}(\mathrm{~T})$ and $\beta_{2}(\mathrm{~T})$ can produce a severe variation in some of the optical parameters evaluated from equations (14) to (17). In order to circumvent this issue, the following procedure for the determination of the molar attenuation coefficients has been employed. A set of molar attenuation coefficients together with initial values of $\Delta S_{d}$ and $\Delta H_{d}$ (i.e. some of those indicated in Table 1 for MB) are used as initial guesses for the error minimization process with the Solver tool in order to minimize the total error $E_{T}$ [for which $\alpha_{1}(T), \alpha_{2}(T), \beta_{1}(T)$ and $\beta_{2}(T)$ parameters are calculated via equations (10) to (13)] under the constrain of positive values of the molar attenuation coefficients. In this way, convergence to very low error values is rapidly attained, and precise values of the molar attenuation coefficients and thermodynamic parameters are finally obtained. The goodness of fit is categorized by both the values of the error $E_{T}$ and the deviation of $K_{d}(T)$ from $K_{d}(A \lambda)$ at the different conditions. 
A chief difference with respect to previous error minimization methods is the form of the error function which rely on experimental and predicted concentration differences [equations (8) and (18)], instead of the common procedure of minimizing the error in absorbance (in [20] both the concentration and absorbance differences are minimized).

For higher MB concentrations (i.e. over $70 \mathrm{ppm}$ [1]), the presence of higher aggregates (i.e. trimers and tetramers) must be accounted for. A more comprehensive method, applicable to higher dye concentrations, will be presented in the near future.

\section{RESULTS AND DISCUSSION}

Figure 1 shows a typical absorption spectrum of MB in the 400-800 nm wavelength range. Linear baselines were automatically evaluated for each spectrum from the minimum absorbance in the 400-450 nm range to the minimum absorbance in the $710-800 \mathrm{~nm}$ range. The values of absorbance at $664\left(A_{\lambda_{1}}\right)$ and $610 \mathrm{~nm}\left(A_{\lambda_{2}}\right)$ were thus obtained after the baseline correction (Fig. 1). The inset in Fig. 1 shows the variation of the $A_{664} / A_{610}$ ratio for the different $\mathrm{MB}$ concentrations and temperatures analyzed. The trends are a clear proof of the dependence of the monomer/dimer ratio on $\mathrm{MB}$ concentration and temperature.

\subsection{Optical and thermodynamic parameters. Refuting the invariant-monomer assumption}

Values of $\varepsilon_{664}^{m}, \varepsilon_{610}^{m}, \varepsilon_{664}^{d}, \varepsilon_{610}^{d}, \Delta S_{d}$ and $\Delta H_{d}$ were evaluated via minimization of the $E_{T}$ error following the procedure above described. The results are indicated in Table 1. The molar attenuation coefficients for the monomer show an appreciable decrease with the increase of temperature for $\lambda_{1}=664 \mathrm{~nm}$ (from $\sim 8 \times 10^{4} \mathrm{~L} \mathrm{~mol}^{-1} \mathrm{~cm}^{-1}$ at $9^{\circ} \mathrm{C}$ to $\sim 7 \times 10^{4} \mathrm{~L} \mathrm{~mol}^{-1} \mathrm{~cm}^{-1}$ at $60^{\circ} \mathrm{C}$ ) whereas the monomer attenuation 
coefficients at $610 \mathrm{~nm}$ show a minute variation and the molar attenuation coefficients for the dimer are invariant regardless the temperature. These values permit the minimization of the $E_{T}$ error to an extremely low value $\left(E_{T}=1.6 \times 10^{-14}\right)$. The low standard deviations of the $K_{d}$ values at the different temperatures can be observed in Table 1 and in the error bars of $K_{d}\left(A_{\lambda}\right)$ included in Figure 2. Also the low $E_{T}$ error may be visualized in the remarkable coincidence of the $K_{d}\left(A_{\lambda}\right)$ data with the $K_{d}(T)$ curve (Fig. 2). As expected, the entropy change is negative due to the association of two similarly charged species [21]. The fraction of monomer can be evaluated at the different conditions by means of the following equation:

$X_{m}=\frac{C_{m}}{C_{M B}}=\frac{\beta_{1} A_{\lambda_{1}}+\beta_{2} A_{\lambda_{2}}}{C_{M B}}$

Figure 3 shows the values of monomer fraction obtained for the different MB concentrations and temperatures. Unsurprisingly, the monomer molar fraction increases with the temperature [9] and with the decrease of MB concentration (equilibrium constant). Finally, the dimer and monomer spectra in the 500-695 nm range were obtained by a simple procedure. The wavelength range was divided into two sectors; at $\lambda>630 \mathrm{~nm}, \lambda_{1}$ was varied between 631 and $695 \mathrm{~nm}$ and $\lambda_{2}$ was fixed at $610 \mathrm{~nm}$, whereas at $\lambda \leq 630 \mathrm{~nm}, \lambda_{1}$ was fixed at $664 \mathrm{~nm}$ and $\lambda_{2}$ was varied between 500 and $630 \mathrm{~nm}$. Then, the $E_{T}$ error was minimized for the different $\left(\lambda_{1}, \lambda_{2}\right)$ couples while keeping the molar attenuation coefficients at 610 and $664 \mathrm{~nm}$ and the thermodynamic parameters $\left(\Delta S_{d}\right.$ and $\left.\Delta H_{d}\right)$ equal to those previously obtained (Table 1$)$. The results of applying this procedure are shown in Figure 4. The upper plot of the figure includes the $K_{d}\left(A_{\lambda}\right)$ and $K_{d}(T)$ values together with the $E_{T}$ value. As can be observed, both the dimeric constant deviations and the errors are very low within almost the whole wavelength range, except in the zone over $\sim 680 \mathrm{~nm}$, where the steep absorbance decrease (Fig. 1) may allow an appreciable variation in the absorbance to be produced by even the slightest deviation in the wavelength. This issue will be further 
analyzed in the last part of this discussion. The spectrum obtained for the dimer displays a profile which greatly differs from those attained by other authors $[6,9]$. It is conspicuous the absence of the maxima at 664 nm obtained by Yazdani et al. [9] and at $695 \mathrm{~nm}$ by Bergmann and O'Konski [6]. Only a maximum at $606 \mathrm{~nm}$ (common to other works) and a decaying shoulder from $\sim 650 \mathrm{~nm}$ are observed in the figure. However, the most striking result appreciated in Figure 4 is the temperature dependence of the monomer spectrum, with a maximum absorbance that decreases at increasing temperatures (Table 1), showing in parallel a small blueshift (from $664 \mathrm{~nm}$ at $9^{\circ} \mathrm{C}$ to $662 \mathrm{~nm}$ at $60^{\circ} \mathrm{C}$ ). This fact proves that the invariant-monomer assumption is incorrect. We believe that this implicit assumption in all previous works is the main source of inaccuracy in both the optical and thermodynamic parameters for MB selfaggregation in water reported in literature (Table 1).

\subsection{Refuting the colorless-monomer assumption}

As mentioned in the Introduction section, Lewis et al. [12] found the same effect in MB spectra obtained at a very low concentration (Table 1 and Figure 5A). The diminution observed in the maximum molar attenuation coefficient with the temperature was attributed to the existence of an additional MB monomer that was assumed to be colorless [12] (colorless-monomer assumption from now on). This species might be the known Leuco-Methylene Blue molecule, which is formed by the reduction of monomeric $\mathrm{MB}$ in the absence of oxygen [19], presenting an absorbance maximum at $\sim 351$ $\mathrm{nm}$, as predicted by TD-DFT calculations [22]. Under the colorless-monomer assumption, the total MB concentration must be evaluated as:

$C_{M B}=C_{m}+C_{c l m}+2 C_{d}$ 
where $C_{c l m}$ stands for the colorless monomer concentration $\left(\mathrm{mol} \mathrm{L}^{-1}\right)$. According to Lewis et al. [12] the colorless monomer is formed via the amphoteric addition of water to the colorful monomer. Thus, the following equilibrium constant, $K_{c l m}$, can be proposed:

$K_{c l m}=\frac{C_{c l m}}{C_{m}}$

This equation results from the obvious consideration that the concentration of water remains essentially unchanged at low $C_{M B}$ values. At a very low $\mathrm{MB}$ concentration, the dimer is practically absent $\left(X_{m} \approx 1\right.$;

Fig. 3) and the Beer-Lambert equation at a $\lambda$ wavelength can be expressed as:

$A_{\lambda}=A_{\lambda}^{m}=\varepsilon_{\lambda}^{m} L C_{m}=\frac{\varepsilon_{\lambda}^{m}}{1+K_{c l m}} L C_{M B}$

Therefore, the observed temperature-dependent molar attenuation coefficients in the absence of dimer can be expressed as:

$\varepsilon_{\lambda}^{m}(T)=\frac{\varepsilon_{\lambda}^{m}}{1+K_{c l m}}$

where $\lambda=660 \mathrm{~nm}$ in the work by Lewis et al. [12]. The data shown in Figure $5 \mathrm{~A}$ are, in principle, consistent with the above equation, provided the $K_{c l m}$ constant increases with the temperature. Lewis $e t$ al. [12] found this trend remarkable, but they still reasoned that it might be possible. On the other hand, as deduced from equation (26), one condition must be accomplished: when the formation of aggregates is discarded, the trend of the temperature-dependent molar attenuation coefficients with the temperature must be a decreasing one for all wavelengths. This condition was checked with different molar attenuation coefficients obtained in this work from the spectra at the lowest concentration $\left(C_{M B}=\right.$ $1.1 \times 10^{-6} \mathrm{~mol} \mathrm{~L}^{-1} ; X_{m}>0.95$; Fig. 3), and it was found that, in many cases, the temperature-dependent coefficients either increased with the temperature or remained more or less constant. Examples of opposite trends are shown in Figure 5B, for wavelengths in the interval 650-690 nm, within which the 
already insignificant effect of the residual dimer concentration is reduced even more (Fig. 4). Therefore, the colorless-monomer assumption is also invalid to explain the different monomer absorption spectra of Figure 4.

Two other alternatives that may explain the temperature-dependent absorption behavior observed in Figures 4 and 5 must be discarded. (i) The little significant MB monomer adsorption on the quartz walls of the cuvette [23] cannot be claimed to be the cause of this behavior, since it would produce the opposite effect; the lower the temperature the higher the MB monomer adsorption and, consequently, the lower the light absorption. (ii) The presence of a mixture of two or more different colorful MB monomers is out of question. The potential presence of a protonated MB monomer, in which the proton is attacking the electron-rich heterocyclic nitrogen [22], would produce a bathochromic effect with the appearance of a peak at $\sim 745 \mathrm{~nm}$ [24], which is absent in all of the spectra obtained in this work. Furthermore, this protonated monomer would be in a significant amount only at very acidic conditions [25]. The blueshift observed on increasing the temperature is certainly too small to be associated to the formation of Bernthsen's methylene violet, the main product from the hydroxyl attack to MB (absorbance maximum at $520 \mathrm{~nm}$ in toluene), which is only produced at basic conditions (pH=13) [22]. Besides, different colorful monomers would potentially produce a variety of dimeric forms with different absorption spectra. In the present work only one absorption spectrum for the dimeric form was needed to perform an accurate fitting to all the absorption profiles.

Therefore, the temperature-dependent absorption behavior of the monomeric form must be ascribed to the presence of a single MB monomer whose molar attenuation coefficient actually changes with the temperature. The following hypothesis raises an explanation about how this can occur. 


\subsection{The resonance virtual equilibrium hypothesis}

The cationic MB molecule is a resonance hybrid with an electronic charge distribution somewhere in between those of the two virtual molecules shown in Figure $6[22,26]$. The mesomer $I$ has a positive charge on the heterocyclic sulphur (the heterocyclic nitrogen is known to bear a negative charge in the ground state [22]), whereas the mesomer II bears the positive charge on the exocyclic nitrogen atom. Figure 6 also shows the direction and values of the theoretical dipole moments in the ground state and in the excited singlet state ( $\mu_{\mathrm{G}}$ and $\mu_{\mathrm{E}}$, respectively) for both mesomers, as reported by Párcányi et al. [27]. The experimental dipole moments in the ground and excited singlet states $\left(\mu_{\mathrm{G}, \mathrm{EXP}}\right.$ and $\mu_{\mathrm{E}, \mathrm{EXP}}$, respectively) have been also evaluated by different methods and are available in the literature [27, 28]. The values indicated in Figure 6 [28] certify a large dipole moment change during transition from the ground state to the excited singlet state, which is due to intermolecular charge transfer and resonance structure contribution. The experimental dipole moment mean value in the excited singlet state (5.0 D) is in good agreement with the theoretical value $(4.7 \mathrm{D})$ calculated with a positive charge on the exocyclic nitrogen. As suggested in [27] this is an indication of a change of distribution of charges in electronically excited states, predicting a migration of the positive charge from the ring sulfur atom to the exocyclic nitrogen atom in the excited singlet state of MB.

The intramolecular charge transfer in the MB molecule is sensitive to the solvent, i.e., it shows solvatochromism [28-30]. However, contrary to the typical betaine-type probes employed in solvatochromic studies [31, 32], the solvatochromism of $\mathrm{MB}$ is poor, that is to say, the solvent polarity has no regular effect on its spectral behavior [28]. In spite of this, Gilani et al. [28] reported a rather obvious bathochromic shift in the $\mathrm{MB}$ absorption spectra for increasing values of solvent dipolarity/polarizability $\left(\pi^{*}\right)$, trend that was also reported in a previous work for all phenothiazines, such as MB, with the exception of thionine [27]. In principle, this fact is consistent with the small 
blueshift observed in Figure 4 at increasing temperatures, since the dielectric constant of water is known to decrease with temperature, from 83.8 at $10^{\circ} \mathrm{C}$ to 63.8 at $70^{\circ} \mathrm{C}[33,34]$. Thus, the observed blueshift can be considered as a type of thermo-solvatochromic effect $[35,36]$. This picture is, however, rather simplistic and does not explain the opposite trends observed in Figure 5B. We believe that the different monomer spectra shown in Figure 4 should not be explained only through thermo-solvatochromic shifts at the wavelength for maximum absorbance, but as the result of the convolution of two fixed absorption spectra whose proportion varies with temperature. And this fact must be reconciled with the existence of a single monomer, that is to say, two spectra for one monomer. To make this possible the resonance virtual equilibrium hypothesis is proposed hereafter. The hypothesis affirms that the temperaturedependent absorption behavior of the monomer is a consequence of a thermo-solvatochromic effect, provoked by the change in the electron charge distribution in the monomer with the variation in the temperature-dependent dielectric constant of water. Within this frame, the main assumptions of the hypothesis are (i) the electron charge distribution of the monomer at a given temperature stands between those of the virtual resonance forms (Figure 6), and (ii) the absorption spectrum of the monomer is a composition of the theoretical spectra for the virtual resonance forms, whose proportion is established by a temperature-dependent equilibrium constant (additivity assumption). Regarding the first assumption, it is known from computational studies that passing from isolated $\mathrm{MB}$ to aqueous (hydrated) MB implies an electronic density transfer in the longitudinal direction from the more external atoms (those bonded to the extremity rings) to the atoms of the central ring of the molecules [37], meaning that the polar environment leads to the stabilization of structures that move away from mesomer I towards mesomer II. Besides, Párcányi et al. [27] indicated that the excited singlet state, which, as commented above, resembles more the mesomer II structure, was more stabilized when the solvent polarity increased. These facts permit to assume that the charge distribution in the MB 
momomer varies in the direction from mesomer II to mesomer I as the temperature increases or, to put it another way, as the water polarity decreases. With respect to the linearity concept implied in the additivity assumption, this is not dissimilar to that made to build the empirical solvent polarity scales [28-30], but in this work it comprises the spectrum in the whole wavelength range analyzed, and not only the wavelength at the maximum absorbance. In any case, the additivity assumption will be either validated or refuted depending on the goodness degree of the fitting procedures performed hereafter.

The virtual equilibrium between mesomeric forms can be defined by the following equilibrium constant:

$$
K_{m}=\frac{C_{m I}}{C_{m I I}}
$$

in which $C_{m I}$ and $C_{m I I}$ must be viewed as the contributions of the charge distributions of mesomers $I$ and II, respectively, to the charge distribution of the actual resonance hybrid (additivity assumption), and not as factual concentrations. The $K_{m}$ equilibrium constant is evaluated as (van't Hoff equation):

$K_{m}(T)=e^{\left(\frac{\Delta S_{m}}{R}-\frac{\Delta H_{m}}{R T}\right)}$

Thus, the monomer concentration can be evaluated as:

$C_{m}=C_{m I}+C_{m I I}=C_{m I I} \times\left(1+K_{m}\right)$

Therefore, following the additivity assumption, at very low MB concentrations equation (25) can be rewritten as:

$A_{\lambda}=A_{\lambda}^{m}=\varepsilon_{\lambda}^{m} L C_{m}=\frac{\varepsilon_{\lambda}^{m I I}+\varepsilon_{\lambda}^{m I} K_{m}}{1+K_{m}} L C_{M B}$

where $\varepsilon_{\lambda}^{m I}$ and $\varepsilon_{\lambda}^{m I I}$ are the theoretical molar attenuation coefficients of mesomers $I$ and $I I$, respectively. In this case the observed temperature-dependent molar attenuation coefficients can be expressed as: 
$\varepsilon_{\lambda}^{m}(T)=\frac{\varepsilon_{\lambda}^{m I I}+\varepsilon_{\lambda}^{m I} K_{m}}{1+K_{m}}$

where $\lambda=660 \mathrm{~nm}$ in the work by Lewis et al. [12] (Figure 5A). Equations (28) and (31) were fitted to the spectra at the lowest concentration $\left(C_{M B}=1.1 \times 10^{-6} \mathrm{~mol} \mathrm{~L}^{-1} ; X_{m}>0.95\right.$; Fig. 3); both the theoretical molar attenuation coefficients for the resonance structures, $\varepsilon_{\lambda}^{m I}$ and $\varepsilon_{\lambda}^{m I I}$, and the thermodynamic parameters $\left(\Delta S_{m}\right.$ and $\left.\Delta H_{m}\right)$ were obtained by minimizing the square differences of the calculated and the experimental values of $\varepsilon_{\lambda}^{m}(T)$. This procedure yielded values for $\Delta S_{m}$ and $\Delta H_{m}$ of $24.0 \mathrm{~J} \mathrm{~mol}^{-1} \mathrm{~K}^{-1}$ and $8.1 \mathrm{~kJ} \mathrm{~mol}^{-1}$, respectively (Table 2). According to the additivity assumption, the $C_{m I} / C_{m I I}$ ratios obtained with the virtual equilibrium constant allow the charge distribution in the monomer to be placed in specific points within the limits established by both resonance forms at the different temperatures; thus, $C_{m I} / C_{m I I}$ varies from 0.58 at $9{ }^{\circ} \mathrm{C}$ to 0.98 at $60^{\circ} \mathrm{C}$, meaning that, in the whole temperature range studied, the charge distribution in the monomer is closer to that of mesomer II than to that of mesomer I. Figure 7A shows the calculated absorption spectra for both mesomers $\left(\varepsilon_{\lambda}^{m I}\right.$ and $\left.\varepsilon_{\lambda}^{m I I}\right)$, whereas Figure 7B reproduces the calculated and experimental spectra at the different temperatures $\left[\varepsilon_{\lambda}^{m}(T)\right]$ for the analyses performed at the lowest $\mathrm{MB}$ concentration. For the sake of clarity, Figures $7 \mathrm{C}$ to $7 \mathrm{E}$ show augmented areas of Figure 7B. The agreement between calculated and experimental plots must be considered remarkable. The continuous curves in Figures 5A and 5B represent the best equation (31) fits to the data reported by Lewis et al. [12] and to the data obtained in this work, respectively, performed in both cases with the same thermodynamic parameters (Table 2). The opposite trends shown in Figure 5B are now reasonably reproduced thanks to the resonance virtual equilibrium hypothesis via equation (31). The values of $\varepsilon_{660}^{m I I}$ and $\varepsilon_{660}^{m I}$ evaluated with this equation are $11.0 \times 10^{4}$ and $4.6 \times 10^{4} \mathrm{~L} \mathrm{~mol}^{-1} \mathrm{~cm}^{-1}$ for the data by Lewis et al. [12] (Figure 5A), and $9.2 \times 10^{4}$ and $5.0 \times 10^{4} \mathrm{~L} \mathrm{~mol}^{-1} \mathrm{~cm}^{-1}$ for the data obtained here (Figure 7A), respectively. The similarity of values is rather acceptable, especially if the following 
sources of uncertainty, which do not affect the values of the thermodynamic parameters, are taken into account: (i) the wavelength accuracy: for instance a small increment in the wavelength would imply a higher $\varepsilon_{660}^{m I I}$ value and a lower $\varepsilon_{660}^{m I}$ value (Fig. 7A), and (ii) the concentration error: low $\mathrm{MB}$ concentrations $(<0.35 \mathrm{ppm})$, that involve smaller precision in the MB weighing or a higher number of dilutions, are more likely to introduce errors in the experimental molar attenuation coefficients than higher MB concentrations. This is why more accurate mesomers' spectra must be re-evaluated from the absorption curves for all the MB concentrations and temperatures, task that can be undertaken by introducing small changes in some of the equations of the method. Under the resonance virtual equilibrium hypothesis equations (10) to (13), (18) and (21) become:

$$
\begin{aligned}
& \alpha_{1}=\frac{-2 \varepsilon_{\lambda_{2}}^{m I I}+\varepsilon_{\lambda_{2}}^{d}+\left(\varepsilon_{\lambda_{2}}^{d}-2 \varepsilon_{\lambda_{2}}^{m I}\right) \times K_{m}}{\left[\left(\varepsilon_{\lambda_{1}}^{m I I} \varepsilon_{\lambda_{2}}^{d}-\varepsilon_{\lambda_{2}}^{m I I} \varepsilon_{\lambda_{1}}^{d}\right)+\left(\varepsilon_{\lambda_{1}}^{m I} \varepsilon_{\lambda_{2}}^{d}-\varepsilon_{\lambda_{2}}^{m I} \varepsilon_{\lambda_{1}}^{d}\right) \times K_{m}\right] L} \\
& \alpha_{2}=\frac{2 \varepsilon_{\lambda_{1}}^{m I}-\varepsilon_{\lambda_{1}}^{d}-\left(\varepsilon_{\lambda_{1}}^{d}-2 \varepsilon_{\lambda_{1}}^{m I}\right) \times K_{m}}{\left[\left(\varepsilon_{\lambda_{1}}^{m I I} \varepsilon_{\lambda_{2}}^{d}-\varepsilon_{\lambda_{2}}^{m I} \varepsilon_{\lambda_{1}}^{d}\right)+\left(\varepsilon_{\lambda_{1}}^{m I} \varepsilon_{\lambda_{2}}^{d}-\varepsilon_{\lambda_{2}}^{m I} \varepsilon_{\lambda_{1}}^{d}\right) \times K_{m}\right] L} \\
& \beta_{1}=\frac{\varepsilon_{\lambda_{2}}^{d}}{\left[\left(\varepsilon_{\lambda_{1}}^{m I I} \varepsilon_{\lambda_{2}}^{d}-\varepsilon_{\lambda_{2}}^{m I I} \varepsilon_{\lambda_{1}}^{d}\right)+\left(\varepsilon_{\lambda_{1}}^{m I} \varepsilon_{\lambda_{2}}^{d}-\varepsilon_{\lambda_{2}}^{m I} \varepsilon_{\lambda_{1}}^{d}\right) \times K_{m}\right] L} \\
& \beta_{2}=\frac{-\varepsilon_{\lambda_{1}}^{d}}{\left[\left(\varepsilon_{\lambda_{1}}^{m I I} \varepsilon_{\lambda_{2}}^{d}-\varepsilon_{\lambda_{2}}^{m I I} \varepsilon_{\lambda_{1}}^{d}\right)+\left(\varepsilon_{\lambda_{1}}^{m I} \varepsilon_{\lambda_{2}}^{d}-\varepsilon_{\lambda_{2}}^{m I} \varepsilon_{\lambda_{1}}^{d}\right) \times K_{m}\right] L} \\
& C_{M B}=\left(\beta_{1} A_{\lambda_{1}}+\beta_{2} A_{\lambda_{2}}\right)\left[1+K_{m}(T)\right]\left[1+2 K_{d}(T) \times\left(\beta_{1} A_{\lambda_{1}}+\beta_{2} A_{\lambda_{2}}\right)\left(1+K_{m}(T)\right)\right] \\
& E_{T}=\frac{1}{N \times M} \sum\left\{C_{M B}-\left[\alpha_{1} A_{\lambda_{1}}+\alpha_{2} A_{\lambda_{2}}+\alpha_{3} A_{\lambda_{3}}\right]\right\}^{2}+ \\
& +\frac{1}{N \times M} \sum\left\{C_{M B}-\left(\beta_{1} A_{\lambda_{1}}+\beta_{2} A_{\lambda_{2}}\right)\left[1+K_{m}(T)\right]\left[1+2 K_{d}(T)\left(\beta_{1} A_{\lambda_{1}}+\beta_{2} A_{\lambda_{2}}\right)\left(1+K_{m}(T)\right)\right]\right\}^{2}
\end{aligned}
$$

All these equations, based on the mass balance $C_{M B}=C_{m}+2 C_{d}=\left(C_{m I}+C_{m I I}\right)+2 C_{d}$, revert back to the former ones if $K_{m}=0\left(C_{m I}=0\right)$. Thus, by maintaining the values of the thermodynamic parameters 
previously evaluated $\left(\Delta S_{d}, \Delta H_{d}, \Delta S_{m}\right.$ and $\Delta H_{m}$; Table 2), the minimization of the $E_{T}$ error [eq. (37)] allows the values of $\varepsilon_{664}^{m I}, \varepsilon_{610}^{m I I}, \varepsilon_{664}^{m I}, \varepsilon_{610}^{m I}, \varepsilon_{664}^{d}, \varepsilon_{610}^{d}$ to be obtained in a first step. Subsequently, the molar attenuation coefficients in the whole wavelength range can be evaluated with the same routine that permitted to build the spectra in Figure 4. The results obtained are shown in Figure SI-1 of the Supporting Information. As displayed in the upper plot of the figure, the dimeric constant deviations and the error values are very low for wavelengths up to $\sim 670 \mathrm{~nm}$, a point from which the errors are clearly magnified. This may be the reason of the apparent discrepancy of the dimer spectrum at $\lambda>670 \mathrm{~nm}$ between Figures 4 and SI-1. As commented before, the sharp absorbance decrease to the right of the maximum (Fig. 1) provokes an appreciable variation in the absorbance by even the slightest deviation in the wavelength (the wavelength accuracy of the Uv-Vis spectrometer used in this work is $\pm 0.3 \mathrm{~nm}$ ). In order to circumvent this issue, the minimization procedure was improved by letting the absorbance at a given wavelength oscillate within the curve formed by the absorbances at $\lambda, \lambda-0.3$ and $\lambda+0.3 \mathrm{~nm}$. The deviation $(\Delta \lambda)$ for each triad of wavelength, MB concentration and temperature was treated as a fitting variable. Figure 8 displays the results. The upper plot shows the wavelength deviations averaged for all MB concentrations and temperatures at each wavelength. With this small correction, it was possible to reach extremely low values both for the $E_{T}$ error and for the $K_{d}$ deviations (middle plot of Figure 8). Finally, the lower plot of Figure 8 shows the refined spectra for the dimer, mesomer I and mesomer II. With the wavelength correction, the zone of the dimer spectrum at $\lambda>670 \mathrm{~nm}$ has now regained the appearance of the spectrum shown in Figure 4. The shapes of the spectra for both mesomers are also similar to the shapes of those previously obtained at the lowest concentration (Fig. 7A), which confirms the validity of the method. Since the spectra in Figure 8 were obtained with all the MB concentration and temperature data, they must be considered more refined than those of Figure 7A. The excellent goodness of fit can be also visualized in the remarkable coincidence between the experimental and 
calculated absorbance curves displayed in Figure SI-2 of the Supporting Information. The molar attenuation coefficients at the maxima of each spectrum of Figure 8, as well as the values of the thermodynamic parameters are indicated in Table 2. It is noteworthy the presence of two maxima in the mesomer I spectrum, at 650 and $681 \mathrm{~nm}$, placed at each side of the maximum for the mesomer II spectrum $(664 \mathrm{~nm})$. This peculiar characteristic of the mesomer I spectrum, which may be the reflection of two different chromophores in this resonance form, is responsible for the change of habit with temperature of the monomer spectra in different wavelength ranges (i.e. Figures 7D and 7E or Figure 5B). In conclusion, the methodology presented in this work together with the resonance virtual equilibrium hypothesis permit the visible light spectroscopic characterization of aqueous MB solutions at low concentration to be established with a high accuracy.

\section{CONCLUSIONS}

After a close examination to the most relevant literature on Methylene Blue self-aggregation in water, a novel procedure based on the Beer-Lambert equation has allowed precise visible-light absorption spectra and thermodynamic equilibrium parameters $\left(\Delta S_{d}=-43.3 \mathrm{~J} \mathrm{~mol}^{-1} \mathrm{~K}^{-1}, \Delta H_{d}=-34.4 \mathrm{~kJ} \mathrm{~mol}^{-1}\right)$ to be obtained for the monomeric and dimeric forms of this cationic dye in water. A thermo-solvatochromic effect detected in the monomer spectra is believed to be the main source of inaccuracy in all previous works. The existence of a colorless form of the monomer, proposed by Lewis et al. [12] to explain the solvatochromic effect, is refuted in this work. In its place, a new hypothesis has been raised; the resonance virtual equilibrium hypothesis. According to this hypothesis the temperature-dependent absorption behavior of the monomer is provoked by the change in its electron charge distribution with the variation in the temperature-dependent dielectric constant of water. The charge distribution stands between those of the virtual resonance forms, being the absorption spectrum of the monomer a 
composition of the theoretical spectra for the virtual mesomers, whose proportion is established by a temperature-dependent equilibrium constant. Thanks to the application of this hypothesis, the virtual equilibrium parameters $\left(\Delta S_{m}=24.0 \mathrm{~J} \mathrm{~mol}^{-1} \mathrm{~K}^{-1}, \Delta H_{m}=8.1 \mathrm{~kJ} \mathrm{~mol}^{-1}\right)$ and molar attenuation coefficients for the mesomers were evaluated with remarkable accuracy.

\section{ACKNOWLEDGMENT}

The financial support for this research work provided by the Spanish MINECO (CTM2014-56770-R project) and FEDER Funds (GRUPIN14-102, Principado de Asturias) is gratefully acknowledged. AFP is grateful to the Spanish MINECO for the award of a contract (BES-2015-072274)

\section{REFERENCES}

[1] D.R. Lemin, T. Vickerstaff, The aggregation of direct dyes and of Methylene Blue 2B in aqueous solution, Transactions of the Faraday Society, 43 (1947) 491-502.

[2] D. Heger, J. Jirkovsk, P. Kln, Aggregation of Methylene Blue in Frozen Aqueous Solutions Studied by Absorption Spectroscopy, The Journal of Physical Chemistry A, 109 (2005) 6702-6709.

[3] E. Braswell, Evidence for trimerization in aqueous solutions of methylene blue, The Journal of Physical Chemistry, 72 (1968) 2477-2483.

[4] Z. Zhao, E.R. Malinowski, Determination of the Hydration of Methylene Blue Aggregates and Their Dissociation Constants Using Visible Spectroscopy, Applied Spectroscopy, 53 (1999) 1567-1574.

[5] Z. Klika, P. Čapková, P. Horáková, M. Valášková, P. Malý, R. Macháň, M. Pospíšil, Composition, structure, and luminescence of montmorillonites saturated with different aggregates of methylene blue, Journal of Colloid and Interface Science, 311 (2007) 14-23.

[6] K. Bergmann, C.T. O'Konski, A spectroscopic study of methylene blue monomer, dimer, and complexes with montmorillonite, The Journal of Physical Chemistry, 67 (1963) 2169-2177.

[7] P.A.R. Tafulo, R.B. Queirós, G. González-Aguilar, On the "concentration-driven" methylene blue dimerization, Spectrochimica Acta Part A: Molecular and Biomolecular Spectroscopy, 73 (2009) 295-300. 
[8] G. Marbán, T.T. Vu, T. Valdés-Solís, A simple visible spectrum deconvolution technique to prevent the artefact induced by the hypsochromic shift from masking the concentration of methylene blue in photodegradation experiments, Applied Catalysis A: General, 402 (2011) 218-223.

[9] O. Yazdani, M. Irandoust, J.B. Ghasemi, S. Hooshmand, Thermodynamic study of the dimerization equilibrium of methylene blue, methylene green and thiazole orange at various surfactant concentrations and different ionic strengths and in mixed solvents by spectral titration and chemometric analysis, Dyes and Pigments, 92 (2012) 1031-1041.

[10] F.W. Weaver, E.L. Quitevis, Relaxation of the methylene blue monomer-dimer equilibrium in supercooled glycerol near the glass transition, Chemical Physics Letters, 378 (2003) 135-141.

[11] E. Rabinowitch, L.F. Epstein, Polymerization of Dyestuffs in Solution. Thionine and Methylene Blue1, Journal of the American Chemical Society, 63 (1941) 69-78.

[12] G.N. Lewis, O. Goldschmid, T.T. Magel, J. Bigeleisen, Dimeric and Other Forms of Methylene Blue: Absorption and Fluorescence of the Pure Monomer, Journal of the American Chemical Society, 65 (1943) 11501154.

[13] K. Ghosh Ashish, A New Variation of Rabinowitch and Epstein's Method of Studying Self-Association of the Dyes: Dimerisation and Trimerisation Constants of Methylene Blue, Zeitschrift für Physikalische Chemie, 94 (1975) 161.

[14] H. Dunken, D. Schmidt, K. Palm, Zur Dimerisation von Methylenblau und Toluidinblau, Zeitschrift für Chemie, 2 (1962) 349-349.

[15] W. Spencer, J.R. Sutter, Kinetic study of the monomer-dimer equilibrium of methylene blue in aqueous solution, The Journal of Physical Chemistry, 83 (1979) 1573-1576.

[16] L. Michaelis, S. Granick, Metachromasy of Basic Dyestuffs, Journal of the American Chemical Society, 67 (1945) 1212-1219.

[17] K. Vetter, J. Bardeleben, Überspannung und Gleichgewichtspotentiale der Methylenblau/Leukomethylenblau-Redoxelektrode, Zeitschrift für Elektrochemie, Berichte der Bunsengesellschaft für physikalische Chemie, 61 (1957) 135-141.

[18] K. Hamada, H. Kubota, A. Ichimura, T. Lijima, S. Amiya, Aggregation of an Azo Dye in Aqueous Solution, Berichte der Bunsengesellschaft für physikalische Chemie, 89 (1985) 859-863.

[19] D. Wales, R. Parker, J. Gates, M. Grossel, P. Smith, Integrated planar Bragg grating oxygen sensor, (2010).

[20] S.M. Ohline, S. Lee, S. Williams, C. Chang, Quantification of methylene blue aggregation on a fused silica surface and resolution of individual absorbance spectra, Chemical Physics Letters, 346 (2001) 9-15.

[21] P. Mukerjee, A.K. Ghosh, The effect of urea on methylene blue, its self-association, and interaction with polyelectrolytes in aqueous solution, The Journal of Physical Chemistry, 67 (1963) 193-197. 
[22] A. Mills, D. Hazafy, J. Parkinson, T. Tuttle, M.G. Hutchings, Effect of alkali on methylene blue (C.I. Basic Blue 9) and other thiazine dyes, Dyes and Pigments, 88 (2011) 149-155.

[23] J. Kalmár, G. Lente, I. Fábián, Kinetics and mechanism of the adsorption of methylene blue from aqueous solution on the surface of a quartz cuvette by on-line UV-Vis spectrophotometry, Dyes and Pigments, 127 (2016) 170-178.

[24] A.K. Ghosh, Study of the self-association of methylene blue from protonation equilibriums, Journal of the American Chemical Society, 92 (1970) 6415-6418.

[25] G.N. Lewis, J. Bigeleisen, Methylene Blue and Other Indicators in General Acids. The Acidity Function, Journal of the American Chemical Society, 65 (1943) 1144-1150.

[26] Z. Li, C.-J. Wang, W.-T. Jiang, Intercalation of Methylene Blue in a High-Charge Calcium Montmorillonite-An Indication of Surface Charge Determination, Adsorption Science \& Technology, 28 (2010) 297-312.

[27] C. Parkanyi, C. Boniface, J. Aaron, M. Maafi, A quantitative study of the effect of solvent on the electronic absorption and fluorescence spectra of substituted phenothiazines: evaluation of their ground and excited singletstate dipole moments, Spectrochimica Acta Part A: Molecular Spectroscopy, 49 (1993) 1715-1725.

[28] A.G. Gilani, M. Salmanpour, T. Ghorbanpour, Solvatochromism, dichroism and excited state dipole moment of azure A and methylene blue, Journal of Molecular Liquids, 179 (2013) 118-123.

[29] L.R.R. Santin, S.C. dos Santos, D.L.R. Novo, D. Bianchini, A.P. Gerola, G. Braga, W. Caetano, L.M. Moreira, E.L. Bastos, A.P. Romani, H.P.M. de Oliveira, Study between solvatochromism and steady-state and time-resolved fluorescence measurements of the Methylene blue in binary mixtures, Dyes and Pigments, 119 (2015) 12-21.

[30] L.M. Moreira, J.P. Lyon, A. Lima, L. Codognoto, D. Severino, M.d.S. Baptista, A.L. Tessaro, A.P. Gerola, N. Hioka, M.R. Rodrigues, J.A. Bonacin, S.C. dos Santos, A.P. Romani, H.P.M. de Oliveira, The Methylene Blue Self-aggregation in Water/Organic Solvent Mixtures: Relationship Between Solvatochromic Properties and Singlet Oxygen Production, 2017.

[31] O.A. El Seoud, Understanding solvation, Pure and Applied Chemistry, 81 (2009) 697-707.

[32] J. Catalán, Toward a Generalized Treatment of the Solvent Effect Based on Four Empirical Scales: Dipolarity (SdP, a New Scale), Polarizability (SP), Acidity (SA), and Basicity (SB) of the Medium, The Journal of Physical Chemistry B, 113 (2009) 5951-5960.

[33] C.G. Malmberg, A.A. Maryott, Dielectric constant of water from $0^{\circ}$ to $100^{\circ} \mathrm{C}$, Journal of research of the National Bureau of Standards, 56 (1956) 1-8.

[34] B.B. Owen, R.C. Miller, C.E. Milner, H.L. Cogan, The dielectric constant of water as a function of temperature and pressure, The Journal of Physical Chemistry, 65 (1961) 2065-2070. 
[35] C.T. Martins, B.M. Sato, O.A.E. Seoud, First Study on the Thermo-Solvatochromism in Aqueous 1-(1Butyl)-3-methylimidazolium Tetrafluoroborate: A Comparison between the Solvation by an Ionic Liquid and by Aqueous Alcohols, The Journal of Physical Chemistry B, 112 (2008) 8330-8339.

[36] E.B. Tada, P.L. Silva, O.A. El Seoud, Thermo-solvatochromism of betaine dyes in aqueous alcohols: explicit consideration of the water-alcohol complex, Journal of Physical Organic Chemistry, 16 (2003) 691-699.

[37] P. Homem-de-Mello, B. Mennucci, J. Tomasi, A. Da Silva, The effects of solvation in the theoretical spectra of cationic dyes, Theoretical Chemistry Accounts, 113 (2005) 274-280. 


\section{Captions to Figures}

Figure 1. Absorption spectrum for an aqueous MB solution. Inset: Variation of $A_{664} / A_{610}$ ratio for the different $\mathrm{MB}$ concentrations and temperatures analyzed in this work

Figure 2. Dimeric equilibrium constants at different temperatures obtained by minimization of the $E_{T}$ error [eq. (21)] for $\lambda_{1}=664 \mathrm{~nm}$ and $\lambda_{2}=610 \mathrm{~nm}$

Figure 3. Monomer fraction at different conditions evaluated by equation (22) for $\lambda_{1}=664 \mathrm{~nm}$ and $\lambda_{2}=610 \mathrm{~nm}$

Figure 4. Upper plot: dimeric equilibrium constants at different temperatures and minimization error [eq. (21)] obtained in the 500-695 nm wavelength range. Lower plot: absorption spectra for the dimer and monomer at different temperatures obtained by minimization of the $E_{T}$ error [eq. (21)]

Figure 5. Symbols: evolution with the temperature at different wavelengths of the observed molar attenuation coefficients in the absence of dimer for (A) data by Lewis et al. [12] $\left(C_{M B}=6.3 \times 10^{-7}\right.$ mol L $\left.{ }^{-1}\right)$ and (B) data obtained in this work $\left(C_{M B}=1.1 \times 10^{-6} \mathrm{~mol} \mathrm{~L}^{-1}\right)$. Solid lines: molar attenuation coefficients predicted by the hypothesis of resonance virtual equilibrium [equation (31)] for $\Delta S_{m}=$ $24.0 \mathrm{~J} \mathrm{~mol}^{-1} \mathrm{~K}^{-1}$ and $\Delta H_{m}=8.1 \mathrm{~kJ} \mathrm{~mol}^{-1}$

Figure 6. Resonance forms of cationic Methylene Blue with values and directions of the theoretical $\left(\mu_{\mathrm{G}}\right.$ and $\left.\mu_{\mathrm{E}}\right)$ and experimental ( $\mu_{\mathrm{G}, \mathrm{EXP}}$ and $\left.\mu_{\mathrm{E}, \mathrm{EXP}}\right)$ moment dipoles for the ground $(\mathrm{G})$ and excited singlet (E) states

Figure 7. A- Molar attenuation coefficients of mesomers $I$ and $I I$ determined by fitting equation (31) to the experimental molar attenuation coefficients obtained at $C_{M B}=1.1 \times 10^{-6} \mathrm{~mol} \mathrm{~L}^{-1}$. B- Experimental molar attenuation coefficients at $C_{M B}=1.1 \times 10^{-6} \mathrm{~mol} \mathrm{~L}^{-1}$ (symbols) and those evaluated by equation (31) 
according to the hypothesis of resonance virtual equilibrium (lines). C to E- Augmented areas of Figure 7B

Figure 8. Upper plot: wavelength deviations employed to minimize the $E_{T}$ error [eq. (37)]. Middle plot: dimeric equilibrium constants at different temperatures and minimization error [eq. (37)] obtained in the 500-695 nm wavelength range. Lower plot: absorption spectra for the dimer, mesomer I and mesomer II obtained by minimization of the $E_{T}$ error [eq. (37)] 


\section{Tables}

Table 1. Literature values of optical and thermodynamic parameters of MB in water at low concentration

\begin{tabular}{|c|c|c|c|c|c|c|c|c|c|c|}
\hline Ref. & $\begin{array}{c}\lambda_{1} \\
\mathbf{n m}\end{array}$ & $\begin{array}{c}\lambda_{2} \\
\mathbf{n m}\end{array}$ & $\varepsilon_{\lambda_{1}}^{m}$ & $\begin{array}{c}\varepsilon_{\lambda_{1}}^{d} \\
\left.10^{-4}\right) \mathrm{L}\end{array}$ & $\begin{array}{c}\varepsilon_{\lambda_{2}}^{m} \\
\mathrm{pol}^{-1} \mathbf{c}\end{array}$ & $\varepsilon_{\lambda_{2}}^{d}$ & $\begin{array}{c}\mathbf{T} \\
{ }^{\circ} \mathbf{C}\end{array}$ & $\begin{array}{l}K_{d} \times 10^{-3} \\
\mathrm{~L} \mathrm{~mol}^{-1}\end{array}$ & $\underset{\mathrm{kJ} \mathrm{mol}^{-1}}{\Delta H_{d}}$ & $\underset{\mathrm{J} \mathrm{mol}^{-1} \mathbf{K}^{-1}}{\Delta S_{d}}$ \\
\hline [11] & 656.5 & 600.0 & 4.00 & 2.04 & 2.07 & 6.28 & 27 & 3.57 & - & - \\
\hline [17] & - & - & - & - & - & - & 25 & 2.59 & - & - \\
\hline$[12]^{\mathrm{a}}$ & 660.0 & - & $\begin{array}{l}8.69^{b} \\
8.44^{b} \\
8.15^{b} \\
7.86^{b}\end{array}$ & $\begin{array}{l}- \\
- \\
- \\
-\end{array}$ & $\begin{array}{l}- \\
- \\
- \\
-\end{array}$ & $\begin{array}{l}- \\
- \\
- \\
-\end{array}$ & $\begin{array}{c}9 \\
23 \\
40 \\
60\end{array}$ & - & - & - \\
\hline [1] & 667.0 & - & 5.83 & - & 2.90 & - & 30 & - & - & - \\
\hline$[13]^{\mathrm{a}}$ & 662.0 & - & 8.50 & - & 3.06 & - & 27 & 2.50 & - & - \\
\hline$[6]^{\mathrm{a}}$ & 664.0 & 605.0 & 9.56 & 2.44 & 3.81 & 13.06 & 25 & $5.88 \pm 0.50$ & - & - \\
\hline [14] & 668.0 & - & 9.00 & - & - & - & $\begin{array}{l}20 \\
30 \\
40 \\
50\end{array}$ & $\begin{array}{l}4.65 \pm 0.28 \\
3.28 \pm 0.20 \\
2.51 \pm 0.15 \\
2.03 \pm 0.13\end{array}$ & $-22.2 \pm 6.3$ & $+4.2 \pm 2.5$ \\
\hline [15] & 660.0 & 610.0 & 7.18 & $1.54^{\mathrm{c}}$ & 3.88 & 9.06 & 20 & $\begin{array}{l}3.97 \pm 0.47^{\mathrm{d}} \\
4.67 \pm 0.52^{\mathrm{e}}\end{array}$ & $-83.2 \pm 10.5$ & $-253.8 \pm 16.7$ \\
\hline$[9]^{\mathrm{a}}$ & 664.0 & 610.0 & 6.61 & 8.94 & 3.02 & 8.18 & $\begin{array}{l}10 \\
20 \\
40 \\
60\end{array}$ & $\begin{array}{c}33.11^{\mathrm{f}} \\
15.49^{\mathrm{f}} \\
3.98^{\mathrm{f}} \\
1.38^{\mathrm{f}} \\
\end{array}$ & $-46.8^{g}$ & $-80.0^{\mathrm{h}}$ \\
\hline $\begin{array}{l}\text { This } \\
\text { work }^{\text {i }}\end{array}$ & 664.0 & 610.0 & $\begin{array}{l}7.98 \\
7.66 \\
7.33 \\
6.98 \\
\end{array}$ & 3.53 & $\begin{array}{l}3.28 \\
3.34 \\
3.33 \\
3.30 \\
\end{array}$ & 10.47 & $\begin{array}{c}9 \\
23 \\
40 \\
60\end{array}$ & $\begin{array}{c}12.68 \pm 0.16^{\mathrm{j}} \\
6.26 \pm 0.09^{\mathrm{j}} \\
2.91 \pm 0.15^{\mathrm{j}} \\
1.31 \pm 0.11^{\mathrm{j}}\end{array}$ & $-34.4^{\mathrm{k}}$ & $-43.3^{k}$ \\
\hline
\end{tabular}

${ }^{\mathrm{a}}$ Molar attenuation coefficients data extracted from figures; ${ }^{\mathrm{b}} C_{M B}=6.3 \times 10^{-7} \mathrm{M} ;{ }^{\mathrm{c}}$ standard deviation $= \pm 3.5 \times 10^{4}$ (very much higher than for the other three coefficients); ${ }^{\mathrm{d}}$ evaluated at $\lambda=610 \mathrm{~nm}$; ${ }^{\mathrm{e}}$ evaluated at $\lambda=660 \mathrm{~nm} ;{ }^{\mathrm{f}} C_{M B}=3.0 \times 10^{-5} \mathrm{M}$; ${ }^{\mathrm{g}}$ average value (from -54.6 to -38.9 ); ${ }^{\mathrm{h}}$ average value (from -104.0 to -56.0 ); ${ }^{\mathrm{i}} E_{T}=1.6 \times 10^{-14}$ [eq. (21)]; ${ }^{\mathrm{j}}$ eq. (19); ${ }^{\mathrm{k}}$ eq. (20)

Table 2. Optical and thermodynamic parameters of MB in water at low concentration

\begin{tabular}{|c|c|c|}
\hline \multicolumn{3}{|c|}{ Optical parameters } \\
\hline Species & $\begin{array}{c}\lambda_{\max } \\
\mathbf{n m}\end{array}$ & $\begin{array}{c}\varepsilon_{\lambda_{\max } \times 10^{-4}} \\
\mathbf{L} \text { mol-1 }^{-1} \mathrm{~cm}^{-1}\end{array}$ \\
\hline Dimer & 606 & 10.45 \\
\hline $\begin{array}{l}\text { Monomer } \\
\text { mesomer II }\end{array}$ & 664 & 10.78 \\
\hline Monomer & 650 & 4.40 \\
\hline mesomer I & 681 & 4.17 \\
\hline
\end{tabular}

\begin{tabular}{|lc|}
\hline \multicolumn{2}{|l|}{ Thermodynamics } \\
\hline Monomer / Dimer \\
$\Delta S_{d}\left(\mathrm{~J} \mathrm{~mol}^{-1} \mathrm{~K}^{-1}\right)$ & -43.3 \\
$\Delta H_{d}\left(\mathrm{~kJ} \mathrm{~mol}^{-1}\right)$ & -34.4 \\
\hline Mesomer II $/$ Mesomer I \\
$\Delta S_{m}\left(\mathrm{~J} \mathrm{~mol}^{-1} \mathrm{~K}^{-1}\right)$ & 24.0 \\
$\Delta H_{m}\left(\mathrm{~kJ} \mathrm{~mol}^{-1}\right)$ & 8.1 \\
\hline
\end{tabular}




\section{Figures}

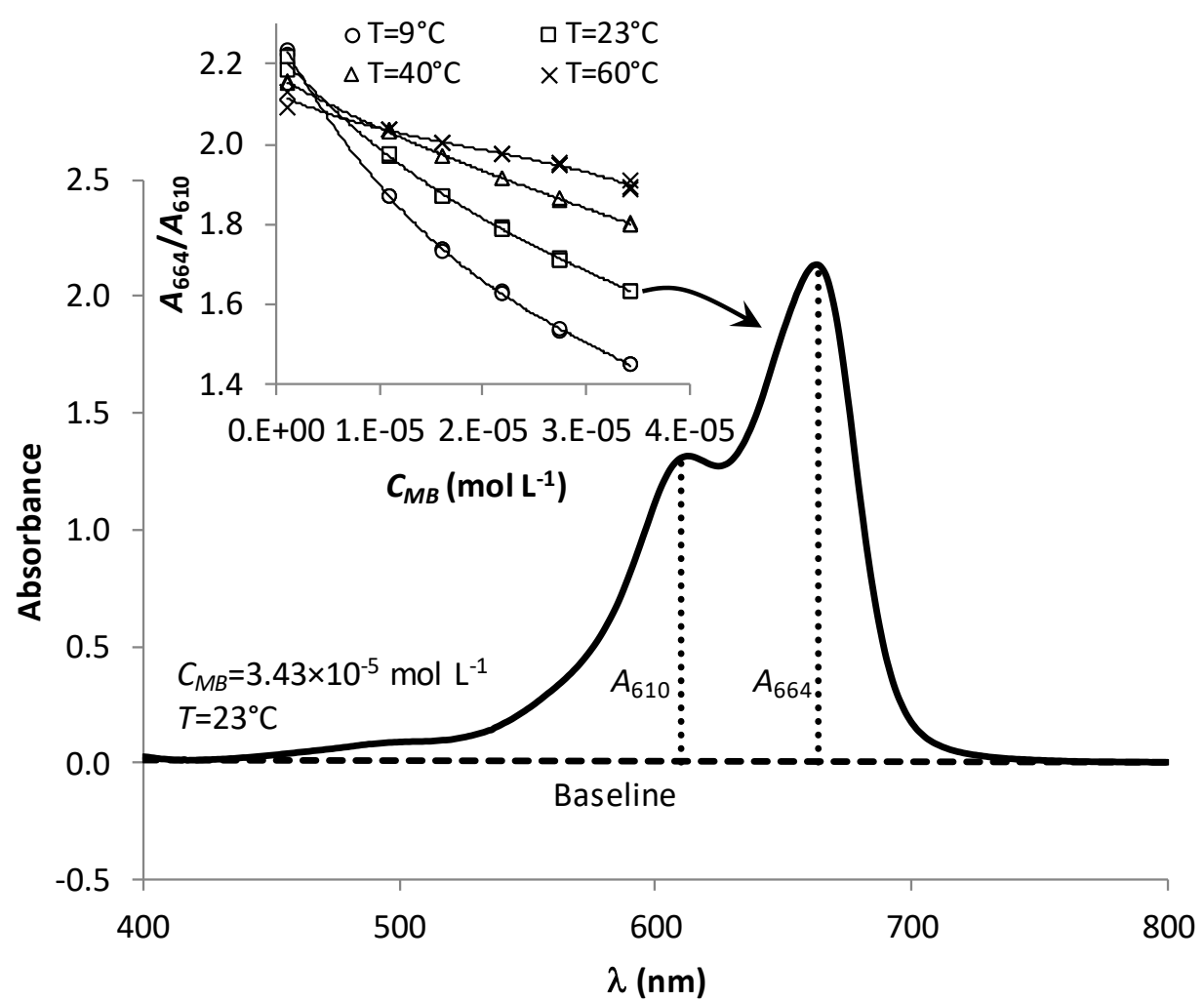

Figure 1 


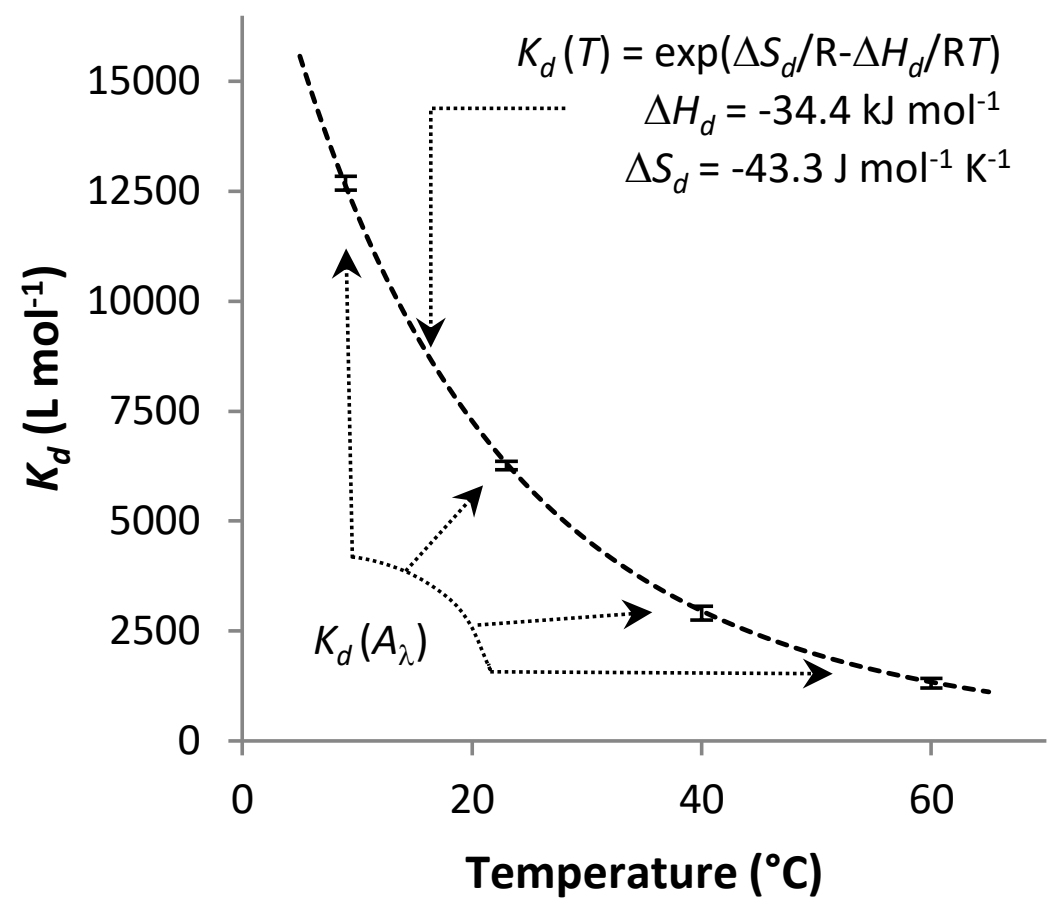

Figure 2 


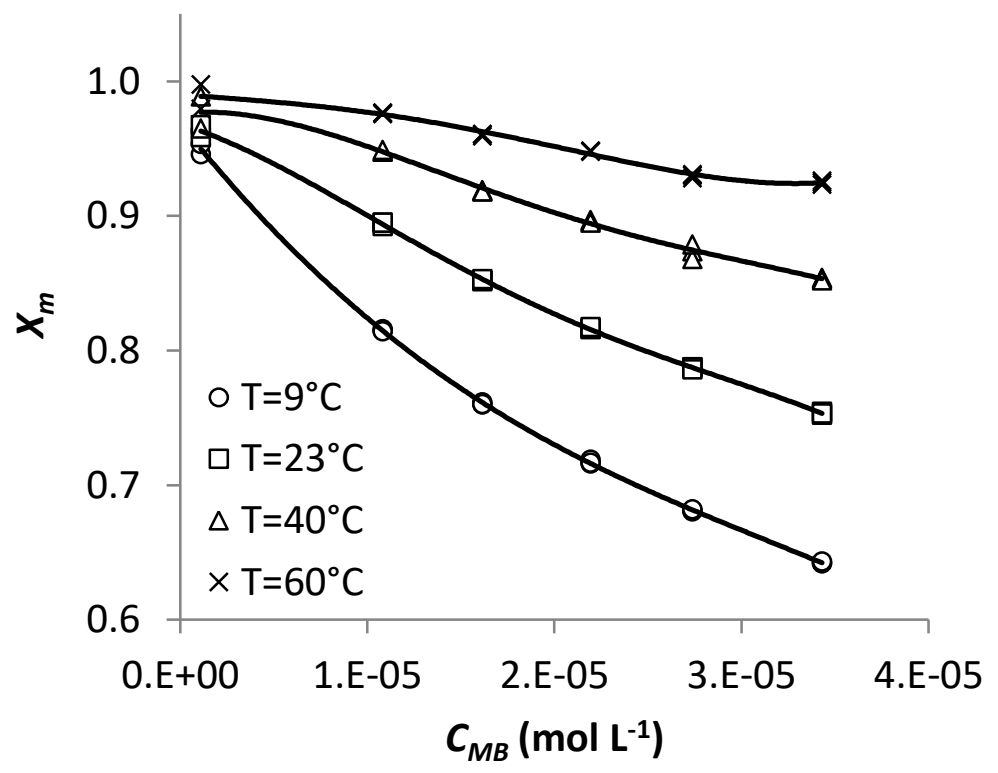

Figure 3 


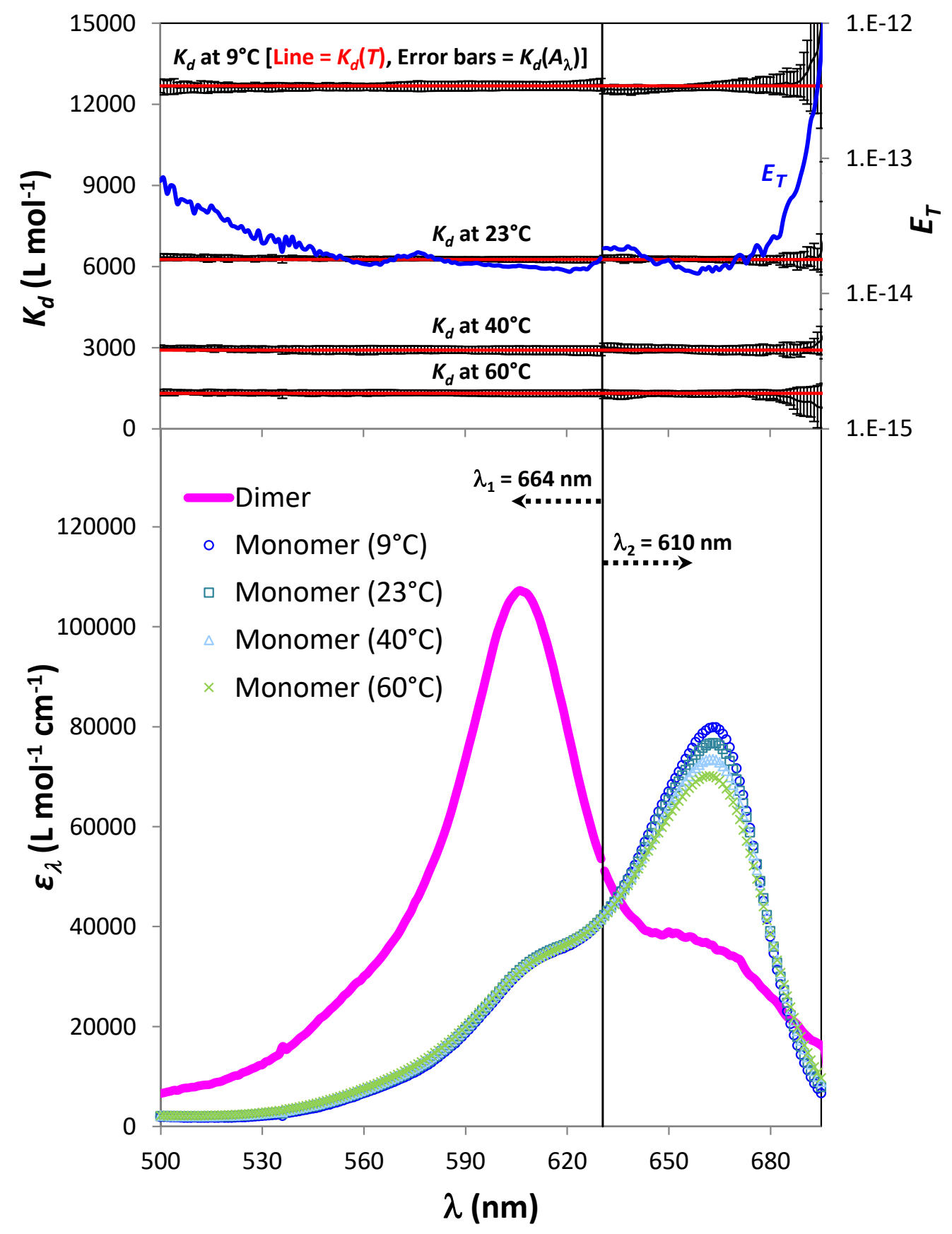

Figure 4 


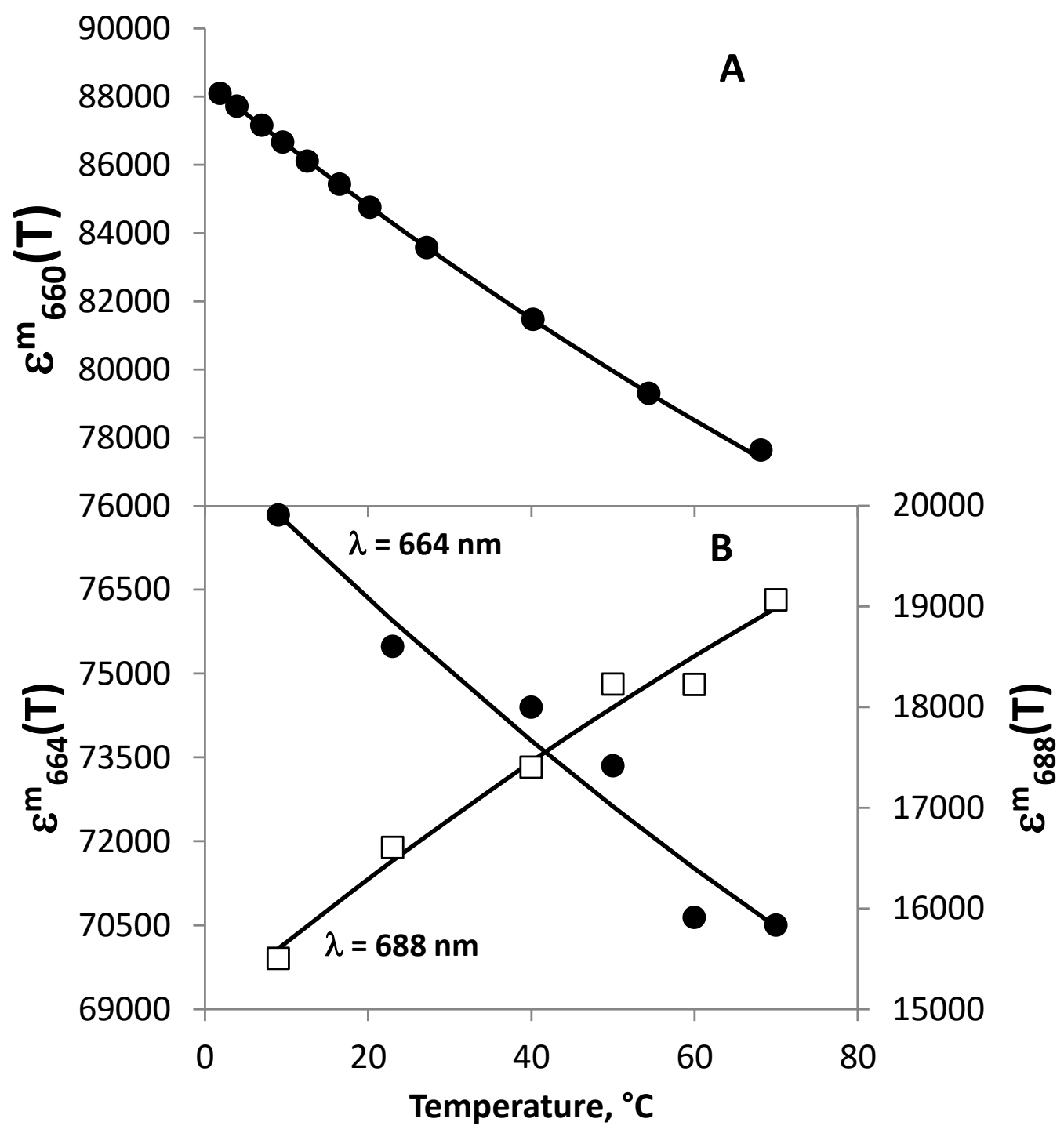

Figure 5 


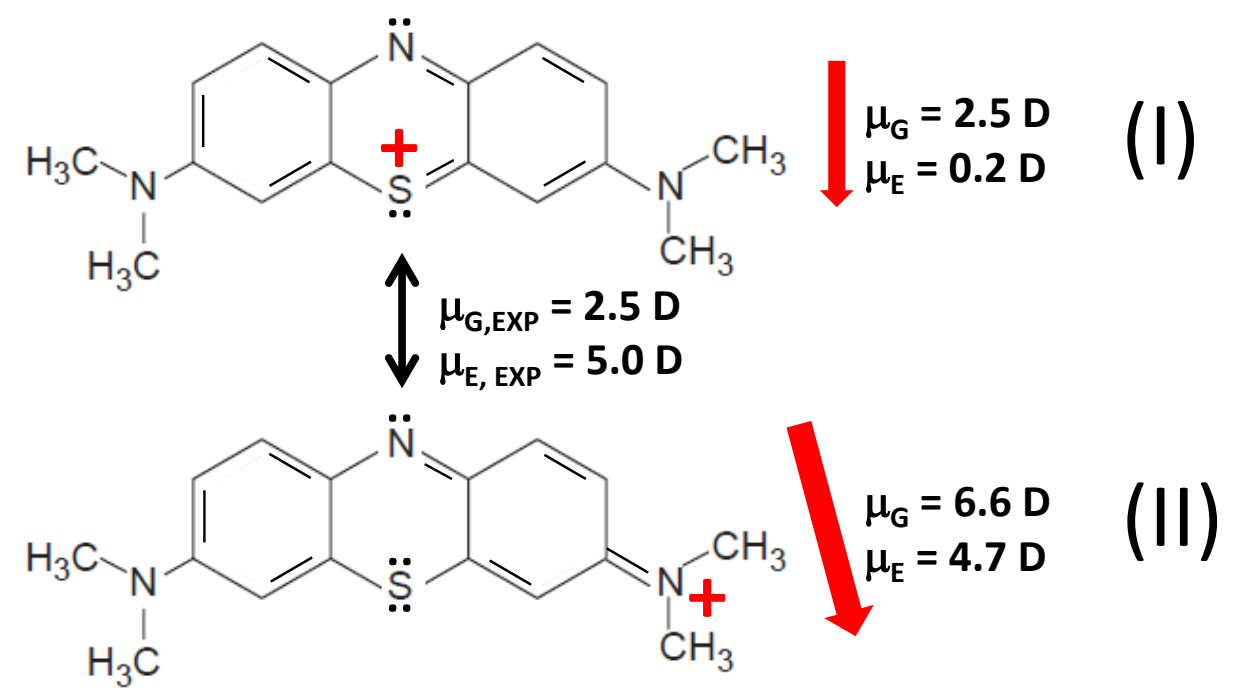

Figure 6 

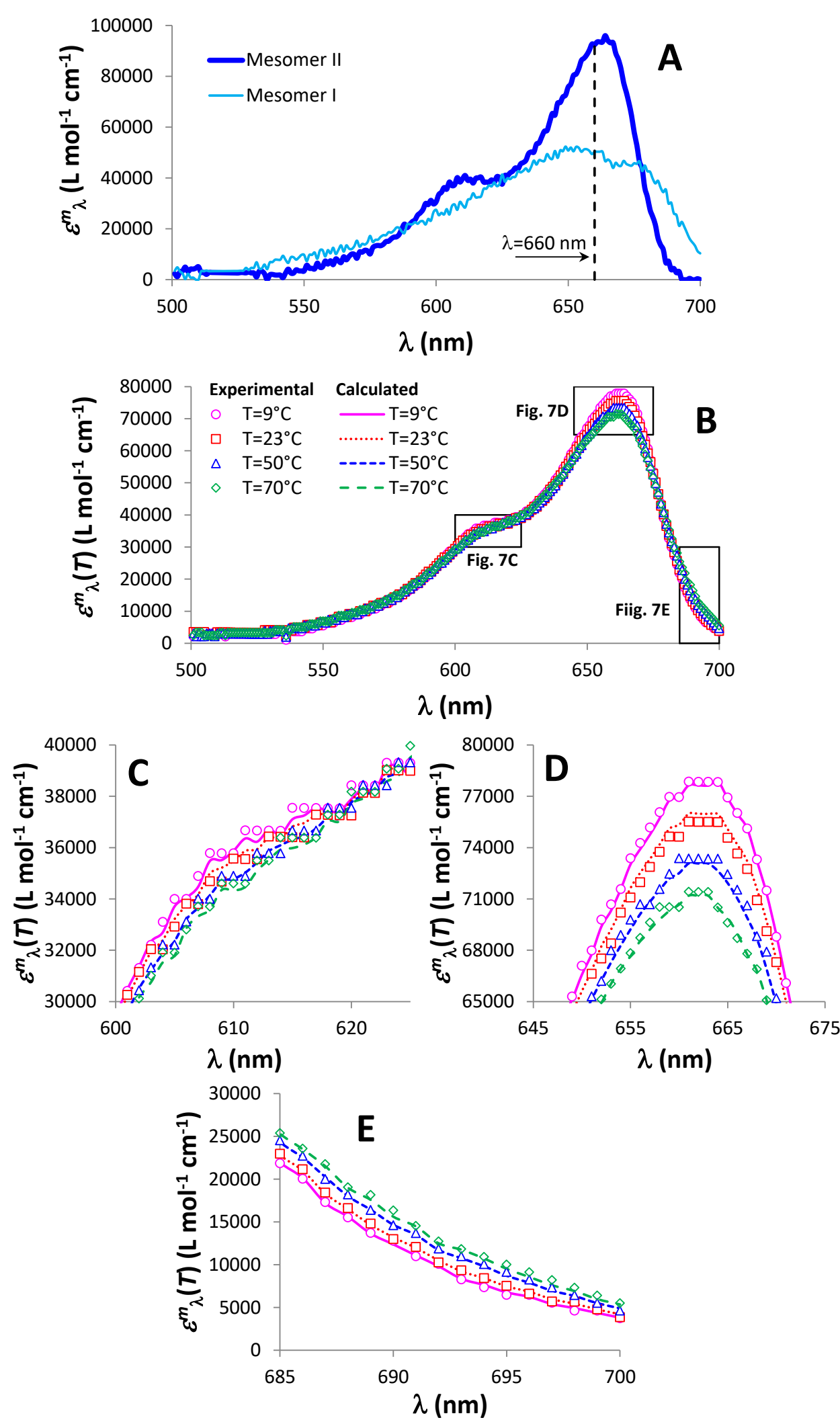

Figure 7 


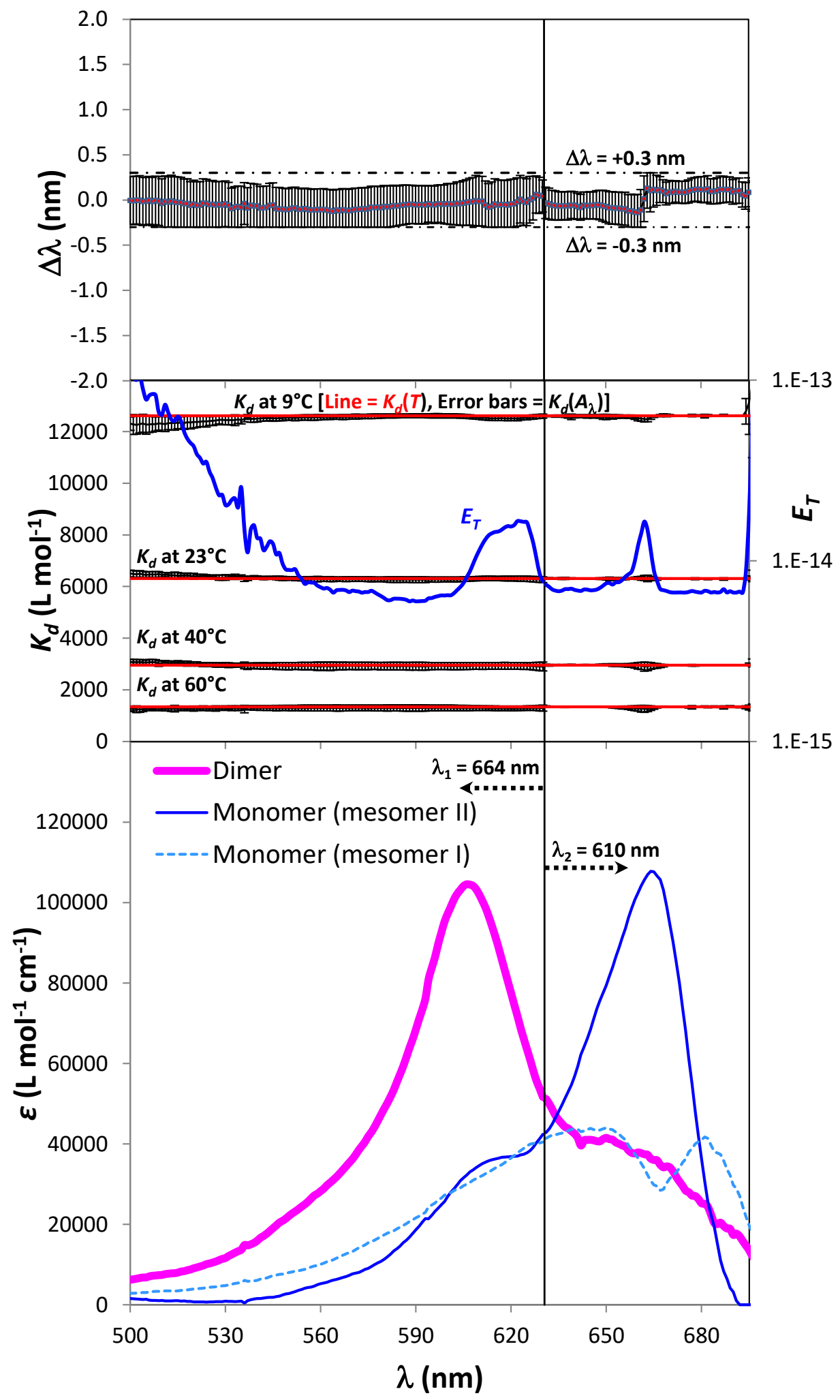

Figure 8 